\title{
Notas sobre os diferenciais educacionais e econômicos da fecundidade no Brasil
}

\author{
Elza S. Berquó ${ }^{*}$ \\ Suzana M. Cavenaghi ${ }^{* *}$
}

\section{Introdução}

No Brasil, assim como em outros países, com a fecundidade em transição ou quase em seu final, a educação e o rendimento se apresentam negativamente correlacionados com o nível da fecundidade. Com a queda desta última, apesar de certa tendência de convergência nas taxas entre os grupos socioeconômicos, observa-se que a fecundidade ainda mantém um diferencial bastante elevado entre as mulheres pertencentes às categorias extremas destes grupos populacionais. Assim, apesar de a taxa de fecundidade de período (TFT) ter alcançado nível abaixo da reposição entre 2000 e 2010, chegando de fato a 1,9 filho por mulher em média, em 2010, conforme dados do Censo Demográfico, não é surpreendente encontrar variações importantes em torno dessa média nacional, diante da diversidade de comportamentos e diferenças no acesso aos meios contraceptivos e de interrupção da gravidez.

Em trabalhos anteriores, Berquó e Cavenaghi (2004; 2005) buscaram quantificar, localizar e qualificar os grupos que já se encontravam com fecundidade abaixo do nível de reposição e aqueles que ainda estavam com taxas de fecundidade elevadas, no período de 1991 a 2000. Para atualizar estas informações e entender as tendências recentes dos diferenciais de fecundidade no Brasil, as autoras desenvolvem o projeto Mapeamento socioeconômico e demográfico dos regimes de fecundidade no Brasil em 2010,1 usando os dados dos Censos Demográficos. No entanto, devido a mudanças importantes no contexto socioeconômico e alteração na informação sobre escolaridade coletada no censo mais recente, algumas decisões metodológicas tiveram que ser consideradas e analisadas.

\footnotetext{
* Centro Brasileiro de Análise e Planejamento (Cebrap), São Paulo-SP, Brasil (berquo@cebrap.org.br).

** Escola Nacional de Ciências Estatísticas (Ence) do Instituto Brasileiro de Geografia e Estatística (IBGE), Rio de Janeiro-RJ, Brasil (suzana_cavenaghi@uol.com.br).

1 Projeto apoiado pelo CNPq, Universal 480541/2012-4.
} 
Neste cenário, o objetivo da presente nota de pesquisa é realizar uma análise das estimativas de fecundidade por grupos educacionais e de rendimento domiciliar per capita em 2000 e 2010, para buscar evidências se o Brasil está diante de uma mudança de comportamento da fecundidade das mulheres mais educadas e mais abastadas ou se, em grande medida, as mudanças observadas devem-se aos efeitos de composição nas estruturas educacionais e de rendimento recentes no país.

\section{Antecedentes}

Os resultados do Censo Demográfico de 2010 trouxeram algumas novidades quanto ao comportamento da fecundidade por grupos etários e segundo categorias de educação e rendimento, principalmente com relação à fecundidade de adolescentes e jovens e, no caso dos diferenciais socioeconômicos, apresentando tendências aparentemente contrárias às que vinham sendo observadas nos resultados dos últimos Censos Demográficos.

Quanto ao comportamento etário, a distribuição da fecundidade recente mostra que as jovens reduziram a taxa específica do grupo de 15 a 19 anos, passando de 93 nascimentos por mil mulheres nessa faixa etária, em 2000, para 71, em 2010, apesar de apresentarem fecundidade ainda relativamente alta, quando comparadas a outros grupos etários e, principalmente, com mulheres residentes em outros países com níveis de fecundidade abaixo do nível de reposição. Assim, o rejuvenescimento da fecundidade, que vinha sendo registrado há várias décadas, já não ocorre e pode até acontecer uma pequena postergação da fecundidade (CAVENAGHI; BERQUÓ, 2014).

Em relação às tendências dos diferenciais de fecundidade por educação e rendimento, os dados mostram que a redução da fecundidade deveu-se, em maior medida, ao comportamento das mulheres menos escolarizadas e mais pobres (CAVENAGHI; BERQUO, 2014), havendo, inclusive, um aparente aumento da taxa para o grupo de mulheres mais escolarizadas. ${ }^{2}$ Entre 2000 e 2010, a TFT passou de 3,7 para 3,0 filhos, entre as mulheres sem instrução ou com até três anos de estudos, e de 2,8 para 2,6 filhos, para aquelas com quatro a oito anos de estudo. De outro lado, as estimativas da TFT mostram, neste mesmo período, aumento de 1,6 para 1,8 filho, entre as mulheres com educação média, de 9 a 11 anos de estudos, e de 1,1 para 1,2 filho, entre aquelas com 12 ou mais anos de estudo.

No que concerne aos diferenciais da fecundidade por rendimento, usando o valor de rendimento médio mensal domiciliar per capita sem considerar a inflação, os resultados analisados pelas autoras mostram que todos os segmentos apresentaram diminuição da taxa de fecundidade, mas a queda é bem maior nos grupos de rendimentos mais baixos. 0 grupo de mulheres em domicílios sem rendimento até um quarto de salário mínimo passou de uma TFT de 4,6 filhos em média, em 2000, para 3,3, em 2010; no outro extremo, para mulheres

\footnotetext{
2 Eduardo Rios-Neto, em apresentação de trabalho no Seminário Internacional Família, Gênero e Gerações - entre mudanças e permanências, realizado de 26 a 27 de novembro de 2013, em Campinas-SP.
} 
em domicílios com cinco ou mais salários mínimos per capita, as taxas corresponderam a 1,2 e 1,1 filho, respectivamente, em 2000 e $2010 .^{3}$

No entanto, estes resultados devem ser avaliados com cuidado, pois a educação e o rendimento são informações coletadas no momento da realização do censo e não no momento do nascimento dos filhos. As mudanças socioeconômicas recentes, portanto, são responsáveis por um efeito de composição destes segmentos populacionais, afetando diretamente o nível de fecundidade do grupo. De fato, atrelado às mudanças demográficas nos últimos anos, principalmente após a virada do século, o Brasil tem passado por um crescimento econômico com mobilidade social ascendente, com diminuição das desigualdades sociais, o que tem permitido a inclusão de amplas parcelas da sociedade no mercado de consumo e ampliação da classe média. O número de domicílios no estrato médio passou de 15,8 milhões, em 1996, para 26,2 milhões, em 2008, segundo dados das PNAD. ${ }^{4}$ Contudo, analisando as distribuições de mulheres de 15 a 49 anos por rendimento médio domiciliar per capita, em 2000 e 2010, verifica-se aumento da porcentagem de mulheres no extremo mais baixo da renda, aquelas em domicílios com até um salário mínimo per capita (de 52\% para 61\%), causando uma compressão dos segmentos com renda média e alta. Isto ocorre devido ao ganho real do salário mínimo do período, além da correção inflacionária que não afeta o rendimento da população que não recebe o correspondente em salários mínimos.

Adicionalmente, nos últimos dez anos, houve uma mudança estrutural muito forte na cobertura educacional no Brasil, principalmente chegando à quase cobertura total no ensino fundamental. Este comportamento se reflete na escolaridade das mulheres de 15 a 49 anos, no período de estudo, e mais fortemente para as mulheres mais jovens. Para dar um exemplo, o número de mulheres com 12 anos ou mais de estudos, que correspondia à metade daquelas com 0 a 3 anos de estudos, apresentou um aumento de quase três vezes no seu contingente, passando de pouco mais de 4 milhões para 10 milhões, entre 2000 e 2010, e ampliando de $9,2 \%$ para $18,8 \%$ sua representatividade relativa no grupo de mulheres de 15 a 49 anos, nesse período (BERQUÓ; CAVENAGHI, 2014). 5

\section{Dados para análise da fecundidade por educação e rendimento}

Apesar de a fecundidade ter alcançado nível abaixo da reposição populacional desde 2005 no Brasil, comparável aos países desenvolvidos, os registros de nascimentos no país ainda não têm cobertura total. Mesmo em locais onde a cobertura dos registros chega

\footnotetext{
3 Os resultados para todos os grupos de rendimento e cruzamento destes com educação podem ser obervados em Cavenaghi e Berquó (2014).

${ }^{4}$ Os motivos citados para a redução da pobreza e das desigualdades sociais no Brasil são muitos, tais como ampliação dos programas de transferência de renda, políticas de valorização do salário mínimo, aumento no número de empregos formais com ganhos reais de renda, avanço no sistema educacional, com a universalização do ensino fundamental e melhorias no ensino médio, e fatores demográficos, como mudanças na composição etária decorrentes da queda da fecundidade (SOARES, 2006; BARROS et al., 2006; IPEA, 2009; NERI, 2009; SOUZA; LAMOUNIER, 2010).

${ }^{5}$ Uma análise mais detalhada das mudanças na estrutura educacional e de rendimento foi apresentada pelas autoras em Berquó e Cavenaghi (2014).
} 
perto do universo de nascimentos, a má qualidade do preenchimento das informações sociodemográficas dificulta sobremaneira o uso dos registros vitais. Dessa forma, a fonte de dados ainda mais recomendada no país para análise dos diferenciais educacionais e de rendimento da fecundidade é o Censo Demográfico. Consequentemente, as estimativas da fecundidade devem ser obtidas por métodos indiretos e, neste estudo, foram comparados o método da razão P/F de Brass (UNITED NATIONS, 1983) e o método relacional de Gompertz (MOULTRIE et al., 2013).

Com relação às variáveis usadas na análise, é importante destacar que, no Censo Demográfico de 2010, não foi incluída a pergunta sobre o último nível de ensino e a última série concluídos com aprovação para as pessoas que não frequentavam a escola no período de referência do censo, impossibilitando o cálculo de anos de estudos completos de forma direta para todas as mulheres (somente permite para aquelas que frequentavam escola no momento da pesquisa). A partir das informações educacionais presentes no questionário do Censo Demográfico de 2010, o IBGE (2013) estimou o nível de instrução para toda a população e disponibilizou essas informações no arquivo de microdados do referido censo. Para o estudo da fecundidade no Brasil, as categorias dos níveis educacionais disponibilizadas não são as mais comumente usadas, pois sabe-se que o ensino fundamental completo tem um limiar importante no comportamento reprodutivo. De outro lado, o ensino superior completo não é compatível com as idades iniciais do ciclo reprodutivo.

Dessa forma, mesmo não sendo possível estimar anos de estudo completo por anos simples, a partir da variável de nível de instrução e demais informações disponíveis no Censo Demográfico de 2010 estimou-se uma nova proxy de categorias educacionais para 2010. Estes grupos educacionais, mais compatíveis com a análise da fecundidade, foram estimados a partir de algoritmo proposto pela Fundação João Pinheiro, ${ }^{6}$ sendo que os grupos de anos de estudo eram: 0 a 3 anos de estudo (sem instrução até primário incompleto); 4 a 8 anos de estudo (primário completo até ensino fundamental completo); 9 a 11 anos de estudo (ensino médio incompleto e completo); e 12 anos ou mais (ensino médio completo e superior incompleto ou completo).

No que concerne à variável rendimento médio domiciliar per capita, alguns procedimentos metodológicos também merecem destaque. O valor em salários mínimos vinha sendo utilizado em trabalhos anteriores pelas autoras, como forma de comparar dados entre os diferentes censos. Devido, principalmente, ao ganho real no salário mínimo, buscaram-se alternativas de comparação. A primeira foi estimar o rendimento em reais equivalentes aos valores de 2000 e, outro, com base nos valores de 2010, todos com base no deflator do INPC, 7 nos dois casos colocados em valores de salário mínimo do ano base. Dessa forma, o cálculo da fecundidade por rendimento médio domiciliar per capita em salários mínimos foi realizado para três tipos de situação: salário mínimo do ano correspondente aos dados

\footnotetext{
${ }^{6}$ O algoritmo utilizado nos microdados da amostra do Censo Demográfico de 2010, a partir da sintaxe do software Statistical Analysis System (SAS), encontra-se em Berquó e Cavenaghi (2014).

${ }^{7}$ Os valores do número índice do INPC foram obtidos no Ipeadata (http://www.ipeadata.gov.br/).
} 
de 2000 e 2010; salário mínimo em valores recebidos em 2000 e inflacionados pelo INPC para 2010, com categorias de SM de 2010; e salário mínimo em valores recebidos em 2010 e deflacionados pelo INPC para 2000, com categorias de SM de 2000.

\section{Métodos de estimativas da fecundidade}

Na América Latina, incluindo o Brasil, e na maior parte dos países com dados de fecundidade incompletos, utilizam-se os métodos indiretos para calcular a fecundidade. Particularmente no Brasil, o método da razão P/F de Brass (UNITED NATIONS, 1983) tem sido amplamente empregado desde a década de 1960 e continua sendo necessária sua aplicação, mesmo estando em nível de fecundidade abaixo do nível de reposição. Recentemente, na revisão do Manual $X$, os autores da versão on-line afirmam que as estimativas das taxas de fecundidade pelo método da razão P/F estão ultrapassadas e o método relacional de Gompertz deveria ser utilizado: "the method in its original and modified forms has been superseded by the relational Gompertz model and its variants" (MOULTRIE et al., 2013, p. 52).

Adicionalmente, os autores afirmam que o método relacional de Gompertz (também proposto inicialmente por Brass) e com solução de Zaba (1981) é um refinamento do método da razão $\mathrm{P} / \mathrm{F}$ de Brass e com algumas vantagens sobre este. Entre tais vantagens, os autores citam que o relacional de Gompertz: não necessita do suposto de fecundidade constante no período mais recente; usa um padrão confiável de regimes de fecundidade média e alta; 8 os dados de cada distribuição linearizada fornecem um guia poderoso para determinar a confiabilidade de cada ponto (grupo etário); e o modelo fornece uma maneira adequada de interpolar os dados em idades simples, permitindo o reagrupamento em grupos etários não convencionais para grupos convencionais (MOULTRIE et al., 2013).

Diante do exposto, foi necessária uma comparação entre os dois métodos para o caso dos dados dos Censos Demográficos, principalmente para grupos socioeconômicos, antes de selecionar o método a ser utilizado na pesquisa. No entanto, de partida este procedimento não seria adequado no caso de estimação de fecundidade para grupos socioeconômicos em que o diferencial de fecundidade é grande entre os grupos, com regimes altos e baixos para diferentes segmentos populacionais, pois seria preciso empregar padrões diferenciados para cada segmento populacional. Além disso, no caso da América Latina, e Brasil, o padrão de fecundidade por idade apresenta-se rejuvenescido para alguns grupos e já mais envelhecido para outros, o que dificulta o uso de um mesmo padrão.

Ainda assim, aplicaram-se ambos os métodos para os dados dos Censos Demográficos de 2000 e 2010 para os grupos educacionais. Os resultados comparando as taxas específicas de fecundidade por idade para os dois métodos são apresentados nos Gráficos 1 e 2. A taxa de fecundidade total é mostrada nas legendas dos gráficos, entre parênteses. O primeiro fato que se observa é que as TFT estimadas, por ambos os métodos, para todos os grupos

\footnotetext{
8 Os autores afirmam que o padrão de fecundidade usado no método não deveria ser empregado para regimes baixos de fecundidade, sendo necessária a estimação de um novo padrão para substituir o padrão utilizado no ajuste proposto no Manual Xon-line.
} 
educacionais, são muito parecidas; as diferenças em geral estão na segunda casa decimal e, mesmo assim, muito próximas. A primeira conclusão decorrente desse resultado é que para a TFT, para ambos os métodos, os resultados são bons, não devendo de antemão se descartar o método da razão $\mathrm{P} / \mathrm{F}$, que tem aplicação mais simples.

O segundo fato decorrente da análise dos Gráficos 1 e 2 é que a taxas específicas de fecundidade por idade (apesar de fornecerem TFT muito similar) apresentam formas diferenciadas para todos os grupos educacionais. Nos grupos de mulheres com menor escolaridade, o método relacional de Gompertz fornece valores mais baixos do que a razão $\mathrm{P} / \mathrm{F}$ para mulheres mais jovens (e obviamente mais altos para mulheres em idades mais avançadas no período reprodutivo). O contrário é observado para os grupos de maior escolaridade, em que o método relacional de Gompertz rejuvenesce a curva de fecundidade, principalmente com maiores níveis de fecundidade para a mulheres de 20-24 e de 25-29 anos, inclusive mudando o pico de fecundidade de 30-34 anos para 25-29, no segmento de mulheres com 12 anos ou mais de estudo.

Em vista dos resultados encontrados 9 e com base no fato de que os dados de fecundidade recente coletados nos Censos Demográficos brasileiros são de boa qualidade (ALVES; CAVENAGHI, 2014), apesar de apresentar a esperada subdeclaração, optou-se por utilizar o método de razão P/F de Brass nesta pesquisa. Adicionalmente, deve-se considerar que o ajuste final selecionado com o método relacional de Gompertz, a despeito de os autores ressaltarem que se podem escolher os melhores conjuntos de pontos na distribuição, depende muito de análise visual, que é bastante trabalhosa, comparada com o método da razão P/F de Brass, que, por sua vez, apresenta resultados mais eficazes para qualquer nível de fecundidade, principalmente, por não impor uma distribuição padrão aos dados.

GRÁFICO 1

Taxas específicas de fecundidade estimadas pelo método da razão $\mathrm{P} / \mathrm{F}$ de Brass e pelo método relacional de Gompertz, por grupos etários, segundo grupos de anos de estudos

Brasil - 2000

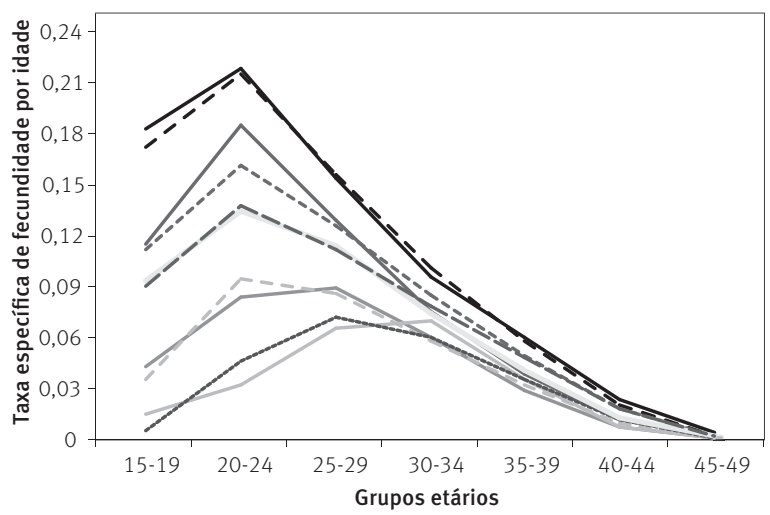

- 0-3 anos de estudo - P/F Brass (TFT 3,69)

- 0-3 anos de estudo - Gompertz (TFT 3,62)

— 4-8 anos de estudo - P/F Brass (TFT 2,79)

- - 4-8 anos de estudo - Gompertz (TFT 2,77)

_ 9-11 anos de estudo - P/F Brass (TFT 1,57)

- - 9-11 anos de estudo - Gompertz (TFT 1,58)

$12 \mathrm{ou}+$ anos de estudo - P/F Brass (TFT 1,13)

-...-. 12 ou + anos de estudo - Gompertz (TFT 1,16)

- Brasil - P/F Brass (TFT 2,36)

- - Brasil - Gompertz (TFT 2,43)

Fonte: IBGE. Censo Demográfico de 2000. Microdados da amostra.

\footnotetext{
${ }^{9}$ Os métodos foram comparados também para os grupos de rendimento domiciliar per capita (não apresentados aqui) e registraram os mesmos resultados por grupos educacionais.
} 
GRÁFICO 2

Taxas específicas de fecundidade estimadas pelo método da razão $\mathrm{P} / \mathrm{F}$ de Brass e pelo método relacional de Gompertz, por grupos etários, segundo grupos de anos de estudos

Brasil - 2010

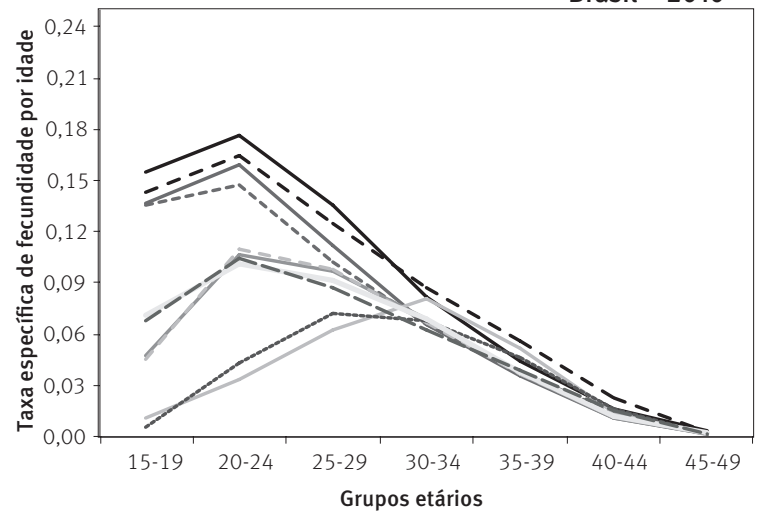

- -3 anos de estudo - P/F Brass (TFT 3,03)

- 0-3 anos de estudo - Gompertz (TFT 2,98)

_ 4-8 anos de estudo - P/F Brass (TFT 2,58)

----4-8 anos de estudo - Gompertz (TFT 2,50)

—-11 anos de estudo - P/F Brass (TFT 1,82)

- - -9-11 anos de estudo - Gompertz (TFT 1,83)

$12 \mathrm{ou}+$ anos de estudo - P/F Brass (TFT 1,26)

....... 12 ou + anos de estudo - Gompertz (TFT 1,25)

Brasil - P/F Brass (TFT 1,90)

- - Brasil - Gompertz (TFT 1,87)

Fonte: IBGE. Censo Demográfico de 2010. Microdados da amostra.

Vale ressaltar ainda que um método paramétrico, que depende de um padrão de fecundidade, deveria ser recomendado somente quando a qualidade dos dados a serem ajustados é de reconhecida má qualidade e, principalmente, apresentam qualidade diferencial por grupos de idade das mulheres, o que não é o caso do Brasil, principalmente nos censos mais recentes (ALVES; CAVENAGHI, 2014).

\section{Os efeitos das mudanças educacionais e de rendimento nas taxas de fecundidade}

Mudanças importantes na estrutura educacional, como a que ocorreu principalmente na década passada, têm a potencialidade de afetar sobremaneira as estimativas de fecundidade de período, como a taxa total de fecundidade, visto que a educação das mulheres é a informação coletada no momento da pesquisa e não no momento que estas mulheres tiveram seus filhos. Deve-se lembrar que a taxa de fecundidade utiliza informação dos filhos nascidos no último ano, portanto, muito provavelmente a mulher tinha a mesma educação declarada ao ter o filho, mas usa também o comportamento da fecundidade acumulada, ou seja, todos os filhos nascidos vivos até o momento da entrevista. Adicionando o padrão jovem da fecundidade do país, com as maiores taxas para mulheres de 20 a 29 e também de 15 a 19 anos, este efeito pode ser ainda maior.

Em situações como essa, o que em geral acontece é que as mulheres que estavam sujeitas a um regime maior de fecundidade, ao melhorarem seu nível educacional, levam consigo uma taxa de fecundidade maior para grupos mais escolarizados. Isso não somente tem o efeito de aumentar a fecundidade desses grupos mais educados, como também de não captar quedas maiores nos grupos menos educados, se seletivamente ficaram nestes grupos as mulheres com maior fecundidade do que aquelas que saíram do grupo. Dados longitudinais, com coleta de informações que variam no tempo, como a escolaridade e o 
número de filhos, é essencial para analisar tendências ao longo do tempo no comportamento da fecundidade. Na ausência destes, o que se pode fazer é mensurar quanto da mudança estrutural educacional (ou outras variáveis) está correlacionado com o nível de fecundidade no período observado.

Um exercício muito simples de padronização da estrutura educacional pode, por exemplo, responder quanto da queda da fecundidade de 2,4 filhos por mulher em média para 1,9 foi devido à mudança na estrutura educacional, assumindo que os quatro grupos de escolaridade utilizados medem adequadamente a estrutura educacional, usando os dados da Tabela 1. Multiplicando-se as taxas de fecundidade de 2010 pela estrutura educacional de 2000, encontra-se o valor de 2,3, ou seja, se a estrutura educacional de 2000 tivesse permanecido em 2010, a fecundidade seria de 2,3 filhos e não de 1,9. Portanto, o efeito da mudança na estrutura educacional está relacionado a uma mudança importante nas taxas de fecundidade, um efeito de $84 \%$ de queda (após controlar pela estrutura etária este feito continua o mesmo).

\section{TABELA 1}

Taxas de fecundidade total e distribuição das mulheres de 15 a 49 anos, segundo grupos de anos de estudos

Brasil - 2000-2010

\begin{tabular}{|c|c|c|c|c|c|c|}
\hline \multirow{3}{*}{$\begin{array}{c}\text { Grupos de } \\
\text { anos de estudo }\end{array}$} & \multicolumn{3}{|c|}{2000} & \multicolumn{3}{|c|}{2010} \\
\hline & \multirow{2}{*}{ TFT } & \multicolumn{2}{|c|}{ Mulheres de 15 a 49 anos } & \multirow{2}{*}{ TFT } & \multicolumn{2}{|c|}{ Mulheres de 15 a 49 anos } \\
\hline & & N. abs. & $\%$ & & N. abs. & $\%$ \\
\hline De 0 a 3 anos & 3,7 & 8.860 .171 & 19,0 & 3,0 & 7.540 .344 & 14,1 \\
\hline De 4 a 8 anos & 2,8 & 20.061 .172 & 42,9 & 2,6 & 14.996 .270 & 28,0 \\
\hline De 9 a 11 anos & 1,6 & 13.125 .451 & 28,1 & 1,8 & 20.922 .408 & 39,1 \\
\hline 12 anos ou mais & 1,1 & 4.279 .854 & 9,2 & 1,2 & 10.065 .866 & 18,8 \\
\hline Total & 2,4 & 46.731.299 & 100,0 & 1,9 & 53.524 .887 & 100,0 \\
\hline
\end{tabular}

Fonte: IBGE, Censos Demográficos de 2000 e 2010. Microdados da Amostra.

As mudanças na estrutura de rendimento são menores do que aquelas observadas na estrutura educacional, mas o ganho real no salário mínimo redistribui as mulheres nos grupos de rendimento com efeitos importantes nos níveis de fecundidade calculados para cada grupo. Usando os valores sem considerar a inflação, os dados da Tabela 2 (colunas 1 e 4) mostram que, ao contrário da educação, o comportamento da TFT para todos os segmentos considerados apresenta diminuição da fecundidade, mas a queda é bem maior nos grupos de rendimento mais baixo. Os grupos de mulheres com maior rendimento, em 2010, são menores do que em 2000, isto é, houve uma "migração" das mulheres dos estratos mais altos para os estratos mais baixos. Por exemplo, embora a população feminina tenha crescido no período, o grupo de mulheres residentes em domicílios com cinco ou mais salários mínimos per capita passou de mais de 4 milhões, em 2000, para somente 2,5 milhões em 2010. Os grupos de mulheres que aumentam em tamanho são aqueles com 1 a 2 salários mínimos ou menos.

A pergunta colocada com os dados da coluna 3 da Tabela 2 é: qual teria sido a taxa de fecundidade em 2000 se a distribuição da renda fosse aquela inflacionada a valores de 
2010? Observa-se que a taxas de fecundidade em 2010 seriam menores ou iguais àquelas estimadas para 2000 em todos os grupos de rendimento. Esse procedimento torna as taxas de 2000 mais comparáveis às de 2010 e mostra, na coluna do número de mulheres em cada segmento, que a mobilidade de renda é ascendente, principalmente para os segmentos acima de meio salário mínimo per capita. Estes segmentos apresentaram uma queda modesta da fecundidade ou fecundidade constante no período. A população feminina com renda mais baixa registrou redução importante da fecundidade para o grupo de zero a $1 / 4$ de salário mínimo, ou seja, ao redor de 15\% da população, passando de 4,1 para 3,3 filhos por mulher.

TABELA 2

Taxas de fecundidade e distribuição das mulheres de 15 a 49 anos, por diferentes cálculos do valor do rendimento, segundo grupos de rendimento médio domiciliar per capita Brasil - 2000-2010

\begin{tabular}{|c|c|c|c|c|c|c|c|c|c|}
\hline \multirow[t]{2}{*}{$\begin{array}{c}\text { Grupos de } \\
\text { rendimento } \\
\text { (em SM de } \\
2000)\end{array}$} & \multicolumn{2}{|c|}{$\begin{array}{c}2000 \\
\text { observado } \\
\text { (1) }\end{array}$} & \multicolumn{2}{|c|}{$\begin{array}{c}2010 \text { equivalente a } \\
2000 \text { (valor de } 2010 \\
\text { levado ao valor de } \\
2000 \text { pelo INPC) } \\
\text { (2) }\end{array}$} & \multirow[t]{2}{*}{$\begin{array}{c}\text { Grupos de } \\
\text { rendimento } \\
\text { (em SM de } \\
2010)\end{array}$} & \multicolumn{2}{|c|}{$\begin{array}{c}2000 \text { equivalente a } \\
2010 \text { (valor de } 2000 \\
\text { levado ao valor de } \\
2010 \text { pelo INPC) } \\
\text { (3) }\end{array}$} & \multicolumn{2}{|c|}{$\begin{array}{c}2010 \\
\text { observado } \\
\text { (4) }\end{array}$} \\
\hline & TFT & $\begin{array}{c}\mathrm{N} . \mathrm{de} \\
\text { mulheres }\end{array}$ & TFT & $\begin{array}{c}\text { N. de } \\
\text { mulheres }\end{array}$ & & TFT & $\begin{array}{c}\text { N. de } \\
\text { mulheres }\end{array}$ & TFT & $\begin{array}{c}\mathrm{N} . \text { de } \\
\text { mulheres }\end{array}$ \\
\hline $\begin{array}{l}\text { Sem } \\
\text { rendimento } \\
\text { e até } 1 / 4 \text { de } S M \\
(\mathrm{R} \$ 0 \text { a } \$ \text { \$ } 37,75)\end{array}$ & 4,6 & 6.532 .303 & 3,1 & 4.987 .885 & $\begin{array}{l}\text { Sem } \\
\text { rendimento e } \\
\text { até } 1 / 4 \text { SM } \\
(\mathrm{R} \$ 0 \text { a } R \$ 127,50)\end{array}$ & 4,1 & 11.233 .088 & 3,3 & 8.270 .383 \\
\hline $\begin{array}{l}1 / 4 \text { a } 1 / 2 \text { SM } \\
(\mathrm{R} \$ 37,75 \text { a } \\
R \$ 75,50)\end{array}$ & 3,2 & 7.086 .790 & 3,6 & 4.474 .308 & $\begin{array}{l}1 / 4 \text { a } 1 / 2 \text { SM } \\
(\mathrm{R} \$ 127,50 \text { a } \\
R \$ 255,50)\end{array}$ & 2,6 & 10.031 .962 & 2,6 & 9.725 .197 \\
\hline $\begin{array}{l}1 / 2 \text { a } 1 S M \\
(\mathrm{R} \$ 75,50 \mathrm{a} \\
\mathrm{R} \$ 151,00)\end{array}$ & 2,4 & 10.577 .392 & 2,5 & 10.759.299 & $\begin{array}{l}1 / 2 \text { a } 1 S M \\
(R \$ 255,50 \text { a } \\
R \$ 510,00)\end{array}$ & 1,9 & 11.062 .800 & 1,9 & 14.554 .981 \\
\hline $\begin{array}{l}1 \text { a } 2 \text { SM } \\
(\mathrm{R} \$ 151,00 \text { a } \\
R \$ 302,00)\end{array}$ & 1,8 & 10.361 .690 & 1,8 & 14.903 .878 & $\begin{array}{l}1 \text { a } 2 \text { SM } \\
(\mathrm{R} \$ 510 \text { a } \\
\mathrm{R} \$ 1.020,00)\end{array}$ & 1,4 & 7.669 .981 & 1,3 & 11.898 .978 \\
\hline $\begin{array}{l}2 \text { a } 3 S M \\
R \$ 302,00 \text { a } \\
R \$ 453,00)\end{array}$ & 1,4 & 4.255 .674 & 1,3 & 7.251 .819 & $\begin{array}{l}2 \text { a } 3 \text { SM } \\
(\mathrm{R} \$ 1.020,00 \text { a } \\
\mathrm{R} \$ 1.530,00)\end{array}$ & 1,3 & 2.642 .021 & 1,1 & 3.800 .550 \\
\hline $\begin{array}{l}3 \text { a } 5 \text { SM } \\
(\mathrm{R} \$ 453,00 \text { a } \\
\mathrm{R} \$ 755,00)\end{array}$ & 1,3 & 3.691 .935 & 1,1 & 5.448 .786 & $\begin{array}{l}3 \text { a } 5 \text { SM } \\
(\mathrm{R} \$ 1.530,00 \text { a } \\
\mathrm{R} \$ 2.550,00)\end{array}$ & 1,2 & 2.104 .763 & 1,1 & 2.756 .906 \\
\hline $\begin{array}{l}5+\mathrm{SM} \\
\text { (Mais de } \\
\mathrm{R} \$ 755,00 \text { ) }\end{array}$ & 1,2 & 4.225 .513 & 1,1 & 5.698 .913 & $\begin{array}{l}5+\mathrm{SM} \\
\text { (Mais de } \\
\mathrm{R} \$ 2.550,00 \text { ) }\end{array}$ & 1,1 & 1.986 .671 & 1,1 & 2.517.891 \\
\hline Total & 2,4 & 46.731 .297 & 1,9 & 53.524 .888 & Total & 2,4 & 46.731 .286 & 1,9 & 53.524 .886 \\
\hline
\end{tabular}

Fonte: IBGE. Censos Demográficos de 2000 e 2010. Microdados da amostra.

Outra comparação que pode ser feita para completar a análise é verificar o que ocorre quando os valores do rendimento em 2010 são deflacionados a valores de 2000 e agrupados conforme o salário mínimo de 2000. Neste caso, o que está implícito é como a população feminina se distribuiria se o salário mínimo tivesse aumentado somente com a correção do INPC, sem o ganho real. Esta comparação é feita entre as colunas 1 e 2 da Tabela 2. Pode-se verificar que, para os grupos de $1 / 4$ a $1 / 2$ SM, por exemplo, a fecundidade parece aumentar 
(de 3,2 para 3,6). No entanto, isso ocorre porque, de fato, este grupo com alta probabilidade recebe salários em equivalência a salário mínimo, o qual aumentou, fazendo, assim, o grupo "migrar" nesta distribuição para segmentos de maior rendimento. As mulheres mais pobres do grupo que realmente ficaram são aquelas com propensão a ter maior fecundidade. No entanto, o grupo de menor rendimento, mesmo com diminuição importante no número de mulheres, ainda mostrou redução da TFT, confirmando que as maiores quedas da fecundidade no período se deram nos grupos mais pobres.

Importante ressaltar que, em 2010, 34\% das mulheres de 15 a 49 anos estavam com fecundidade bem acima da reposição, cerca de $27 \%$ encontravam-se na média nacional (e perto do nível de reposição) e quase $40 \%$ apresentavam fecundidade superbaixa (inferior a 1,3 filho). Este último grupo era composto de mulheres com rendimento médio domiciliar per capita de um ou mais salários mínimos. O que ocorrerá com a economia nos próximos anos pode ser determinante daquilo que vai acontecer com a taxa de fecundidade no país. É muito provável que a diminuição da fecundidade de jovens e das mais pobres continue, mas, por outro lado, parece improvável que a maioria da população alcance níveis econômicos com mais de um salário mínimo per capita em um futuro próximo.

\section{Considerações finais}

A análise das tendências da taxa de fecundidade ao longo dos anos, para segmentos socioeconômicos da população, em cenário de mudanças no quadro educacional e econômico é uma tarefa bastante difícil. Nesta etapa do trabalho, buscou-se analisar os efeitos das mudanças ocorridas na estrutura educacional nas taxas de fecundidade e como a mobilidade econômica ascendente também afeta essas taxas.

Para realizar o estudo, procurou-se, em primeiro lugar, definir qual o melhor método de estimar a fecundidade a ser aplicado no caso dos censos brasileiros e para os recortes populacionais do estudo. Chegou-se à conclusão de que o método da razão P/F de Brass ainda é o mais conveniente a ser empregado. Outros desenvolvimentos metodológicos deveriam ser realizados para que o método relacional de Gompertz pudesse ser aplicado.

Com relação às tendências da fecundidade na última década, conclui-se que a queda da fecundidade ocorreu em maior medida entre as mulheres mais pobres, diminuindo ainda mais os diferenciais socioeconômicos. Também verificou-se que esta queda se deu em maior medida devido às mudanças na estrutura da educação e do rendimento. As mulheres melhoraram sua educação e ascenderam para estratos de melhor rendimento e, com isso, a fecundidade das mais educadas e das mais abastadas aparentemente se manteve constante ou apresentou um leve aumento.

O conhecimento detalhado das taxas de fecundidade, em relação tanto ao nível quanto à estrutura por idade da fecundidade, em diversos grupos socioeconômicos, é extremamente importante para a definição de cenários futuros para a projeção da fecundidade, que é o componente demográfico que mais afeta as projeções e estimativas populacionais. Estas, por 
sua vez, são medidas essenciais para o planejamento de políticas públicas, principalmente aquelas que necessitam de intervenção local. Dessa forma, a pesquisa busca contribuir para a melhoria das previsões de cenários futuros para a taxa de fecundidade no país e para segmentos sociais importantes. Caso a educação continue em processo de avanço, bem como a melhora no rendimento da população, espera-se que a fecundidade diminua ainda mais nos próximos anos, mas tudo dependerá muito do futuro quadro econômico e educacional no país.

\section{Referências}

ALVES, J. E. D; CAVENAGHI, S. Informações sobre fecundidade a partir do Censo Demográfico: ponderações metodológicas e as influências do tipo de informante. In: VI CONGRESSO DA ASSOCIAÇÃO LATINO AMERICANA DE POPULAÇÃO. Anais... Lima, Peru, 2014.

BARROS, R. P.; CARVALHO, M.; FRANCO, S.; MENDONÇA, R. S. P. Uma análise das principais causas da queda recente da desigualdade de renda brasileira. Econômica, v. 8, n. 1, p. 117-147, jul. 2006.

BERQUÓ, E. S.; CAVENAGHI, S. M. Brazilian fertility regimes: profiles of women below and above replacement levels. In: XXV INTERNATIONAL CONFERENCE IUSSP. Anais... Tours, França: IUSSP, 2005.

Mapeamento sócio-econômico e demográfico dos regimes de fecundidade no Brasil e sua variação entre 1991 e 2000. In: XIV ENCONTRO NACIONAL DE ESTUDOS POPULACIONAIS. Anais... Belo Horizonte: Abep, 2004.

. Tendências dos diferenciais educacionais e econômicos da fecundidade no Brasil entre 2000 e 2010. In: XIX ENCONTRO NACIONAL DE ESTUDOS POPULACIONAIS. Anais... São Pedro/SP: Abep, 2014.

CAVENAGHI, S.; BERQUO, E. Perfil socioeconômico e demográfico da fecundidade no Brasil de 2000 a 2010. In: VI CONGRESSO DA ASSOCIAÇÃO LATINO AMERICANA DE POPULAÇÃO. Anais... Lima, Peru, 2014.

CAVENAGHI, S; ALVES, J. E. D. Diversity of childbearing behaviour within population in the context of below replacement fertility in Brazil. United Nations Expert Group Meeting on Recent and Future Trends in Fertility. Population Division, United Nations Department of Social and Economic Affairs. New York, 2009. Disponível em: 〈www.un.org/esa /population/meetings/...Fertility2009/P10_Cavenaghi.pdf〉.

IBGE. Metodologia do Censo Demográfico 2010. Série Relatórios Metodológicos, v.41, 2013, p. 713. Disponível em: «http://servicodados.ibge.gov.br/Download/Download.ashx? http=1\&u= biblioteca.ibge. gov.br/visualizacao/livros/liv81634.pdf`. Acesso em: 22 set. 2014.

IPEA. Trajetória recente da mudança na identidade e na estrutura social brasileira. Comunicado da Presidência, n. 34, novembro de 2009. Disponivel em: 〈http://www.ipea.gov.br/default.jsp〉.

MOULTRIE, T. A. et al. Tools for demographic estimation. Paris: International Union for the Scientific Study of Population, 2013. Disponivel em: 〈http://demographicestimation.iussp.org〉. Acesso em: 13 jan. 2014.

NERI, M. Crônica de uma crise anunciada. Choques externos e a nova classe média. Rio de Janeiro, FGV, 2009.

SOARES, S. S. D. Análise de bem-estar e decomposição por fatores da queda na desigualdade entre 1995 e 2004. Econômica, v. 8, n. 1, p. 83-115, 2006. 
SOUZA, A.; LAMOUNIER, B. A. Classe média brasileira: ambições, valores e projetos de sociedade. Rio de Janeiro: Ed. Campus, 2010.

UNITED NATIONS. Manual X: indirect techniques for the demographic estimation. Population Studies, 81. New York: National Research Council, United States National Academy of Sciences, 1983.

ZABA, B. Use of the relational Gompertz model in analysing fertility data collected in retrospective surveys. London: Centre for Population Studies, London School of Hygiene \& Tropical Medicine, 1981 (Working paper, 81-2).

\section{Sobre as autoras}

Elza Berquó é doutora em Bioestatística pela Columbia University. Professora Emérita da Faculdade de Saúde Pública da Universidade de São Paulo, 2014. Título de Pesquisador Emérito do CNPq, 2013, membro da Ordem do Mérito Científico Classe Grã-Cruz, 1998, e membro titular da Academia Brasileira de Ciências, 2000.

Suzana Cavenaghi é doutora em demografia pela Universidade do Texas-Austin e professora e pesquisadora da Escola Nacional de Ciências Estatísticas (Ence) do Instituto Brasileiro de Geografia e Estatística (IBGE), atuando no Programa de Pós-Graduação e População, Território e Estatísticas Públicas.

\section{Endereço para correspondência}

Escola Nacional de Ciências Estatísticas

Rua André Cavalcanti, 106, sala 502, Bairro de Fátima

20231-050 - Rio de Janeiro-RJ

Recebido para publicação em 03/11/2014

Aprovado para publicação em 12/12/2014 